\title{
Octonionic Gravity Formation, Its Connections to Micro Physics
}

\author{
Andrew Beckwith \\ Department of Physics, Chongqing University, Chongqing, China \\ E-mail:abeckwith@uh.edu \\ Received March 14, 2011; revised May 1, 2011; accepted May 11, 2011
}

\begin{abstract}
We ask if Octonionic quantum gravity is a relevant consideration near the Planck scale. Furthermore, we examine whether gravitational waves would be generated during the initial phase, $\delta_{0}$, of the universe when triggered by changes in spacetime geometry; i.e. what role would an increase in degrees of freedom have in setting the conditions during $\delta_{0}$, so that the result of these conditions can be observed and analyzed by a gravitational detector. The micro physics interaction is due to the formation of a pre Planckian to Planckian space time transition in spatial dimensions at and near the Planck dimensional values, i.e. $10^{-33}$ centimeters in spatial dimensions. This transition would be abrupt and arising in micro physics regimes of space time.
\end{abstract}

Keywords: High-Frequency Gravitational Waves (HFGW), Symmetry, Causal Discontinuity

\section{Introduction}

The Planck epoch has remained mysterious, and may be invisible to all other kinds of detectors, but the universe's gravity wave background radiation likely contains the imprint of even the very earliest events. Changes in the geometry of space-time near the Planck scale could be revealed or studied in this manner. We discuss how to obtain insights into $\delta_{0}$, initially, while looking at the geometric considerations determining space and time development which would create relevant space-time geometry phase changes during the early universe. The formation of Planckian space time is in what is called Octonionic quantum gravity [1] Each such phase change leading to Octonionic Quantum gravity should produce gravitational waves. The geometry change alluded to is effectively initially on an infinitesimal scale, which is why Micro physics is referred to at all. ('quantum mechanics') and de facto quantum gravity, at the start of Planckian space time. This Planckian space time would mark the beginning of inflation. We give conditions for detection of [2,3] $\delta_{0}$ if, for example, one can isolate an appropriate first-order perturbative electromagnetic power flux, [2-4] $T^{u v}$ in scenarios where the graviton has a vanishingly small - but non-zero - rest mass [2]. Now for how to get non zero graviton rest mass. This is where the micro physics regime becomes so important.
The Micro Physics Problem. How to Solve the Correspondence Problem, I. E. (Gravitons in 4D with Slight Mass (5 Degrees of Freedom) Versus Gravitons in 4 D with NO Mass (2 Degrees of Freedom).

This formation of a graviton, must be done before the formation of QM, in space time.

The correspondence problem, is that a Graviton with slight rest mass in $4 \mathrm{D}$ will have five degrees of freedom wheras a Graviton in $4 \mathrm{D}$, with no mass has two degrees of freedom. I.e. there is no way about this, and that if gravitons as brought up by Beckwith [2] have a small rest mass, which contributes to DE speed up of acceleration of the universe a billion years ago, gravitons cannot be synthesized during the time when QM becomes dominant. This document gives a regime of space time before QM, which embeds quantum mechanics. If a $4 \mathrm{D}$ graviton with rest mass not equal to zero is formed, it will be in the template of space time before to up to $10^{-44}$ seconds. I.e. as stated, so as to have GW/Gravitons with rest mass not equal to zero forming [2], The document mentions a phase alteration, which we claim is due to this alteration from classical embedding of quantum mechanics, to its full expression in the Planckian space time regime. This micro physics leads to [2-4] $\delta_{0}$

What we propose is the following evolution in the micro physics regime, up to $10^{-44}$ seconds

1) That the degrees of freedom increase, with an in- 
crease in temperature, during a transition to a Rindler Geometry flat space regime of space time. As given in Equation (16), with increasing temperature, more degrees of freedom unfold from a topological transition. Degrees of freedom likely approach a maxima as temperature does, but this is a subject needing experimental exploration and verification.

2) That for low but non zero initial temperature, the so called cold universe model, in pre space time in the pre Planckian regime, one has initially a huge degree of generated entropy. At the same time, we have about 2 degrees of freedom, with complex geometry in each geometrical slot, geoinfometric instantiation, or "infometron" of space time, which large quantities of stored entropy enveloped in the 'crevices' between infometrons, or lattice points.

3) Low degrees of freedom for low temperature corresponds to a complex geometry storing large amounts of total entropy in a complex geometric structure, and that later the entropy is released, with a break down of this complex geometric structure, i.e. equivalent to having many lattices, highly ordered, with low degrees of freedom per 'lattice', to many degrees of freedom (DOF) as space time 'lattices' are broken, releasing entropy. The analogy is not perfect, but approximates what would happen as one goes from complex curved space geometry with many 'crevices' for storage of entropy, which are released, "apparently" leading to a lot of entropy,.

Further elaboration of what is being brought up is tied in with mutually unbiased basis (MUB), [5] . The values of $\delta_{0}$ are set by the difference between Renyi entropy [6], and a particle count version of entropy, i.e. $\mathrm{S} \sim\langle n\rangle$. Are predictions also possible regarding signal strength of evolutionary artifacts of early universe HFGW? Again, yes. What we are talking about is the break down, due to thermal heat flux of an initial mutually unbiased basis set for a very complex initial geometry, and a reconstitution of space time geometry in flat Euclidian space time regime. The topological transition is due to a change in basis / geometry from the regime of Renyi entropy [6] to entropy in a particle count version of entropy, i.e. $\mathrm{S} \sim\langle n\rangle$. The choice of a Gaussian mapping, with two variable inputs, as given by Equation (16) below is done as a simplest case model. We will model inputs into the initial value of $\Lambda$ as high energy fluctuations, and see if they contribute to examination of the formation of non commutative geometry in the beginning/just before the inflationary era.

\subsection{First, Thermal Input into the New Universe. In Terms of Vacuum Energy}

We will briefly allude to temperature drivers which may say something about how thermal energy will be introduced into the onset of a universe. This will be the 'thermal driver' for the increase in degrees of freedom. Begin first with looking at different value of the cosmological vacuum energy parameters, in four and five dimensions [7]

$$
\left|\Lambda_{5-\operatorname{dim}}\right| \approx c_{1} \cdot\left(1 / T^{\alpha}\right)
$$

in contrast with the more traditional four-dimensional version of the same, minus the minus sign of the brane world theory version. as given by Park [8]

$$
\Lambda_{4-\operatorname{dim}} \approx c_{2} \cdot T^{\beta}
$$

Right after the gravitons are released, one still sees a drop-off of temperature contributions to the cosmological constant. Then for time values $t \approx \delta^{1} \cdot t_{P}, 0<\delta^{1} \leq 1$ and integer $\mathrm{n}$ [7]

$$
\frac{\Lambda_{4-\operatorname{dim}}}{\left|\Lambda_{5-\operatorname{dim}}\right|}-1 \approx \frac{1}{n}
$$

In terms of its import the following has been suggested in the initial phases of the big bang, with large vacuum energy $\neq \infty$ and $a\left(t^{*}\right) \neq 0,0<a\left(t^{*}\right) \ll 1$, the following relation, which violates (signal) causality, is obtained for small fluctuation $a\left(t^{*}\right)<l_{P}$ If we examine $\left|\Lambda_{5-\operatorname{dim}}\right| \sim c_{2} T^{-\beta}$

We assume in this that we have, a discontinuity in the pre Planckian regime, for scale factors [7].

$$
\left[\frac{a\left(t^{*}+\delta t\right)}{a\left(t^{*}\right)}\right]-1<(\text { value }) \approx \varepsilon^{+} \ll 1
$$

Furthermore, in the transition for $0 \leq t<t_{P}$ the following increase in degrees of freedom is driven by thermal energy from a prior universe starting with [9]

$$
E_{\text {thermal }} \approx \frac{1}{2} k_{B} T_{\text {temperature }} \propto\left\lfloor\Omega_{0} \breve{T}\right\rfloor \sim \breve{\beta}
$$

The assumption is that there is an initial fixed entropy arising, with $\bar{N}$ as a nucleated structure in short time interval as temperature $T_{\text {temperature }} \varepsilon\left(0^{+}, 10^{19} \mathrm{GeV}\right)$ arrives.

If the inputs into the inflaton $\phi$, as given by a random influx of thermal energy from temperature, we will see the particle count on the right hand side of Equation (13)

$$
[\Delta S]=[\hbar / T] \cdot\left[2 k^{2}-\frac{1}{\eta^{2}}\left[M_{\text {Planck }}^{2} \cdot\left[\left[\frac{6}{4 \pi}-\frac{12}{4 \pi}\right] \cdot\left[\frac{1}{\phi}\right]^{2}-\frac{6}{4 \pi} \cdot\left[\frac{1}{\phi^{2}}\right]\right]\right]\right]
$$


above a random creation of $n_{\text {Particle-Count }}$. The way to introduce the expansion of the degrees of freedom from nearly zero to having $\mathrm{N}(\mathrm{T}) \sim 10^{3}$ is to define the classical and quantum regimes of gravity as to minimize the point of the bifurcation diagram affected by quantum processes [9]. Dynamical systems modeling is employed right 'after' evolution through the 'quantum dot' regime. The diagram, would look like an application of the Gauss mapping of [9].

$$
x_{i+1}=\exp \left[-\tilde{\alpha} \cdot x_{i}^{2}\right]+\tilde{\beta}
$$

The inputs of change of iterated steps on the right hand side of Equation (14) may indeed show increase in degrees of freedom. Change of temperature, as given, over a short distance, is $[5,6]$

$$
\begin{aligned}
& \frac{\Delta \tilde{\beta}}{\text { dist }} \cong\left(5 k_{B} \Delta T_{\text {temp }} / 2\right) \cdot \frac{\bar{N}}{\text { dist }} \sim q E_{\text {net-electric-field }} \\
& \sim \text { change in degree of freedom }
\end{aligned}
$$

We would regard this as being the regime in which we see a thermal increase in temperature, up to the Planckian physics regime. If so, then we can next look at what is the feeding in mechanism from the end of a universe, or universes, and inputs into Equation (7), Equation (8).

\subsection{Formal Proof that Increase in Thermal Temperatures as Given in Table 1 Leads to Approaching Quantum Mechanics, As an End Run about the Correspondence Problem.}

To do this we look at [1] as to how to look at the way we may have, if temperatures increase, as stated in Table 1 above, from a low point to a higher one, for there to be a flattening of space time and the end of non communative geometry. This non communative geometry due to rising temperatures signifies conditions for the emergence of Equation (23) to become [1]

$$
\left[x_{j}, p_{i}\right] \underset{\operatorname{Temp} \rightarrow \infty}{\longrightarrow} i \hbar \delta_{i, j}
$$

In order to get conditions for Equation (24) the following can be referred to about non commutative geometry [1]

Table 1. Cosmological $\Lambda$ in 5 and 4 dimensions [7], with emphasis upon the Micro physics initial regime. $0 \leq t<t_{P}$ (PLANCK TIME $\sim 10^{-44}$ SECONDS).

\begin{tabular}{lll}
\hline Time $0 \leq t \ll t_{P}$ & Time $0 \leq t<t_{P}$ & Time $t \geq t_{P}$ \\
\hline$\left|\Lambda_{5}\right|$ undefined, & $\left|\Lambda_{5}\right| \approx \varepsilon^{+}$, & $\left|\Lambda_{5}\right| \approx \Lambda_{4-\text { dim }}$, \\
$T \approx \varepsilon^{+} \rightarrow T \approx 10^{32} K$ & $\Lambda_{4-\text { dim }} \approx$ extremely & $T$ much smaller \\
$\Lambda_{4-\text { dim }} \approx$ almost $\infty$ & large & than $T \approx 10^{12} K$ \\
\hline
\end{tabular}

$$
\left[x_{j}, x_{i}\right]=i \cdot\left[\Theta_{j i}\right] \underset{T e m p \rightarrow \infty}{\longrightarrow} 0
$$

The essentials step is to say the anti symmetric real tensor is proportional to the square of 1 over the Parks representation of the "Planck constant", which has a temperature dependence built in it [1].

$$
\Theta_{j i} \sim \Lambda_{N C}^{-2} \sim\left[\Lambda_{4-D i m}\right]^{-2} \propto 1 /\left[T^{2 \beta}\right] \underset{T \rightarrow \infty}{\longrightarrow} 0
$$

When Equation (26) goes to zero, leading to Equation (10) going to zero, we submit that then Equation (10) is recovering quantum/Octonian gravity. The Equation (24) is linkable to initial violations of Lorentz invariance. We submit that the entire argument of Equation (22) to Equation (25), as given by Equation (11) with rising temperature is a way to understand the removal of non Euclidian space to approach Euclidian flat space. We shall next examine how this increasing temperature may lead to an explosion of the degrees of freedom present.

\subsection{Understanding how Phase Shift in \\ Gravitational Waves may be Affected by the Transition to and from a Causal Discontinuity, and Different Models of Emergent Structure Cosmology}

We will outline research initiated by . Beckwith and Li, and Yang Nang, gives us details of gravitational wave generation by early universe conditions. In [3] as given by Li, and Yang, 2009, Beckwith [2] outlined in Chongquing the following representation of amplitude, i.e. as by reading [3] the following case for amplitude

$$
A_{\otimes}=A_{\oplus}=\breve{A}
$$

Furthermore, the first order perturbative (E and $M)$ terms of an E\&M field may have its components written as [3]

$$
\tilde{F}_{02}^{(1)}=i \tilde{F}_{0}^{(1)}
$$

Secondly, there is a way to represent the" number" of transverse first order perturbative photon flux density as given by (in an earth bound high frequency $\mathrm{GW}$ detector). [3]

$$
\begin{gathered}
n_{r}^{(1)}=\frac{c}{2 \mu_{0} \hbar \omega_{e^{-}}} \operatorname{Re}\{\} \\
\{\}=i(\exp [-i \theta]) \cdot \tilde{F}_{0}^{(1)^{*}} \cdot\left[\frac{i}{\omega_{e^{-}}} \cdot\left(\frac{\partial \Psi_{x}}{\partial y}-\frac{\partial \Psi_{y}}{\partial x}\right)\right]
\end{gathered}
$$

Here the quantity $\frac{i}{\omega_{e^{-}}} \cdot\left(\frac{\partial \Psi_{x}}{\partial y}-\frac{\partial \Psi_{y}}{\partial x}\right)$ represents the $z$ component of the magnetic field of a Gaussian beam to be used in an EM cavity to detect GW. We introduce the 
quantity $Q$, the quality factor of the detector cavity set up to observe GW, and $\breve{A}$, the experimental GW amplitude. In the simplest case, $\hat{B}_{y}^{(0)}$ is a static magnetic field. Then the $\tilde{F}_{0}^{(1)}=i \tilde{F}_{0}^{(1)}$ will lead to $[2,3]$

$$
\tilde{F}_{0}^{(1)}=i 2 \breve{A} \hat{B}_{y}^{(0)} Q \cdot\left[\sin \left[\frac{n \pi z}{b}\right]\right] \cdot \exp \left[i-\omega_{g} t+\delta_{0}\right]
$$

The formula $E_{\text {thermal }} \approx \frac{1}{2} k_{B} T_{\text {temperature }} \propto \quad \breve{\beta}$ is a feed into $\omega_{g}$ provided that we pick time $t \propto$ Planck time, and set Equation (16) with $\omega_{g} \sim \omega_{g}$ by setting up the $E_{\text {thermal }} \approx \frac{1}{2} k_{B} T_{\text {temperature }} \approx \breve{\beta} \quad$ [9]. In other words, for relic $\mathrm{GW} /$ graviton production, a topological transformation and interrelationship between $\tilde{\alpha}$ and

$E_{\text {thermal }} \approx \frac{1}{2} k_{B} T_{\text {temperature }} \propto \breve{\beta}$ for increases in (topological) degrees of freedom, as a change in geometry, i.e. before quantum gravity. Passing gravitons through to a new universe is not the same thing though as a pre Planckian geometry, for Octonian gravity conditions arise in early Planckian space time. This is a different perspective than what is normally used in analyzing what happens in a transition between initial Planck time $10^{-44}$ seconds, and cosmological evolution up to $10^{-30}$ seconds We will specify how to locate massive gravitons, via an experimental set up which may enable obtaining data for supporting a value [2,3] for $\delta_{0}$. The next discussion is on research done by Dr. F. Li, et al., 2003, [4],

\section{Re Casting the Problem of GW/Graviton in a Detector for "Massive" Gravitons}

We now turn to the problem of detection. The following discussion is based upon with the work of Dr. Li, Dr/ Beckwith, and other Institute of theoretical physics researchers in Chongquing University [2-4]. It is now time to consider what happens if one is looking for traces of gravitons which may have a small rest mass in four dimensions. What Li et al. have shown in 2003 [2-4] which Beckwith commented upon and made an extension in [2] is to obtain a way to present what is called here in the literature $[3,4] \mathrm{a}$ first order perturbative electromagnetic power flux, i.e. what was called $T^{u v}$ in terms of a non zero four dimensional graviton rest mass, in a detector, in the presence of uniform magnetic field, when examining the following situation, i.e. [2-4] what if we have curved space time with say an energy momentum tensor of the electro magnetic fields in GW fields as given by

$$
T^{u v}=\frac{1}{\mu_{0}} \cdot\left[-F_{\alpha}^{\mu} F^{v \alpha}+\frac{1}{4} \cdot g^{\mu v} F_{\alpha \beta} F^{\alpha \beta}\right]
$$

Li et al. $[3,4]$ state that $F_{\mu v}=F_{\mu v}^{(0)}+\tilde{F}_{\mu v}^{(1)}$, with $\left|\tilde{F}_{\mu v}^{(1)}\right| \ll\left|F_{\mu v}^{(0)}\right|$ will lead to

$$
T^{u v}=T^{(0)}+{ }^{u v}+T^{u v}+T^{u v}
$$

The $1^{\text {st }}$ term to the right hand side of Equation (18) is the energy - momentum tensor of the back ground electro magnetic field, and the $2^{\text {nd }}$ term to the right hand side of Equation (18) is the first order perturbation of an electro magnetic field due to the presence of gravitational waves. The above Equation (17) and Equation (18) will eventually lead to a curved space version of the Maxwell equations as [3]

$$
\frac{1}{\sqrt{-g}} \cdot \frac{\partial}{\partial x^{v}} \cdot\left(\sqrt{-g} \cdot g^{\mu \alpha} g^{v \beta} F_{\alpha \beta}\right)=\mu_{0} J^{\mu}
$$

as well as

$$
F_{[\mu v, \alpha]}=0
$$

Eventually, with GW affecting the above two equations, we have a way to isolate $T^{u v}$. If one looks at if a four dimensional graviton with a very small rest mass included [2-4] we can write[2] an extension of Li et al. $[3,4]$.

$$
\frac{1}{\sqrt{-g}} \cdot \frac{\partial}{\partial x^{v}} \cdot\left(\sqrt{-g} \cdot g^{\mu \alpha} g^{\nu \beta} F_{\alpha \beta}\right)=\mu_{0} J^{\mu}+J_{\text {effective }}
$$

where for $\varepsilon^{+} \neq 0$ but very small $[3,4]$

$$
F_{[\mu v, \alpha]} \sim \varepsilon^{+}
$$

The claim which A. Beckwith made [2] is that

$$
J_{\text {effective }} \cong n_{\text {count }} \cdot m_{4-D-\text { Gravition }}
$$

As stated by Beckwith, in [2],

$m_{4-D-\text { Gravition }} \sim 10^{-65}$ grams, while $n_{\text {count }}$ is the number of gravitons which may be in the detector sample. What Beckwith, Li, and Chonquing university researchers intend to do is to try to isolate out an appropriate $T^{u v}$ assuming a non zero graviton rest mass, and using Equation (21), Equation (22) and Equation (23). From there, the energy density order contributions of, $T^{u v}$ i.e. $T^{(1)}$ can be isolated, and reviewed in order to obtain traces of $\breve{\beta}$, which can be used to interpret Equation (14). I.e. use $\breve{\beta} \cong|F|$ and make a linkage of sorts with $T^{00}$. The term $T^{(1)}$ isolated out from $T^{(1)}$ present day data. The point here that the detected GW would help constrain and validate Equation (14). If this is done, the next step will be to come up with a protocol as far as making sense out of different GW measurement protocols.

Working with a unified $h_{0}^{2} \Omega_{G W}$. The value picked for 
general measurements was

$$
h_{0}^{2} \Omega_{G W} \sim 10^{-6}
$$

Next, after we tabulate results with this measurement standard, we will commence to note the difference and the variances from using $h_{0}^{2} \Omega_{G W} \sim 10^{-6}$ as a unified measurement which will be in the different models discussed right afterwards

\subsection{Wavelength, Sensitivity and other such Constructions from Maggiore, with our Adaptations and Comments}

We will next give several of our basic considerations as to early universe geometry which we think are appropriate as to Maggiore's [10] treatment of both wavelength, strain, and $\Omega_{G W}$ among other things. As far as early universe geometry and what we may be able to observe, such considerations are make or break as to the role of early universe geometry and the generation of GW at the start of the universe. To begin with, we will look at Maggiore's [10] $\Omega_{G W}$ formulation, his ideas of strain. The idea will be to look at how the ten to the tenth stretch out of generated wave length may tie in with early universe models. We will from there proceed to look at, and speculate how the presented conclusions factor in with information exchange between different universes.

We begin with the following tables, Table 1 and Table 2 . The idea will be to, if one has $h_{0}=0.51 \pm 0.14$, as a degree of measurement uncertainty begin as to understand what may be affecting an expansion of the wave lengths of pre Planckian GW/gravitons which are then increased up to ten orders of magnitude. What we have stated below in Table 2 will have major consequences as far as not only information flow from a prior to present universe, but also fine tuning the degree of $\mathrm{GW}$ variance

What we are expecting, as given to us by L. Crowell, is that initial waves, synthesized in the initial part of the Planckian regime would have about $\lambda_{G W} \sim 10^{-14}$ meters for $f_{G W} \sim 10^{22}$ Hertz which would turn into $\lambda_{G W} \sim 10^{-1}$ meters, for $f_{G W} \sim 10^{9}$ Hertz, and sensitivity

Table 2. Managing GW generation from pre planckian physics and its immediate aftermath.

\begin{tabular}{lll}
\hline$h_{C} \leq 2.82 \times 10^{-33}$ & $f_{G W} \sim 10^{12}$ Hertz & $\lambda_{G W} \sim 10^{-4}$ meters \\
\hline$h_{C} \leq 2.82 \times 10^{-31}$ & $f_{G W} \sim 10^{10}$ Hertz & $\lambda_{G W} \sim 10^{-2}$ meters \\
$h_{C} \leq 2.82 \times 10^{-29}$ & $f_{G W} \sim 10^{8} \mathrm{Hertz}$ & $\lambda_{G W} \sim 10^{0}$ meters \\
$h_{C} \leq 2.82 \times 10^{-27}$ & $f_{G W} \sim 10^{6} \mathrm{Hertz}$ & $\lambda_{G W} \sim 10^{2}$ meters \\
$h_{C} \leq 2.82 \times 10^{-25}$ & $f_{G W} \sim 10^{4} \mathrm{Hertz}$ & $\lambda_{G W} \sim 10^{1}$ kilometer \\
$h_{C} \leq 2.82 \times 10^{-23}$ & $f_{G W} \sim 10^{2} \mathrm{Hertz}$ & $\lambda_{G W} \sim 10^{3}$ kilometer \\
\hline
\end{tabular}

of $h_{C} \leq 2.82 \times 10^{-30}$. This is assuming that $h_{0}^{2} \Omega_{G W}$ $\sim 10^{-6}$, using Maggiorie's [10] $h_{0}^{2} \Omega_{G W}$ analytical expression. It is important to note in all of this, that when we discuss the different models that the $h_{0}^{2} \Omega_{G W} \sim 10^{-6}$ is the first measurement metric which is drastically altered. $h_{C}$ which is mentioned in Equation (26) should be also noted to be an upper bound. In reality, only the $2^{\text {nd }}$ and $3^{\text {rd }}$ columns in Table 1 above escape being seriously off and very different., since the interactions of gravitational waves/gravitons with quark - gluon plasmas and even neutrinos would serve to deform by at least an order of magnitude $h_{C}$. So for Table 1, the first column is meant to be an upper bound which, even if using Equation (40.c) may be off by an order of magnitude. More seriously, the number of gravitons per unit volume of phase space as estimated, is heavily dependent upon $h_{0}^{2} \Omega_{G W} \sim 10^{-6}$.

The particle per phase state count will be given as, if $h_{0}^{2} \Omega_{G W} \sim 10^{-6} \quad[10]$

$$
n_{f} \sim h_{0}^{2} \Omega_{G W} \cdot \frac{10^{37}}{3.6} \cdot\left[\frac{1000 H z}{f}\right]^{4}
$$

Secondly we have that a detector strain for device physics is given by [10]

$$
h_{C} \leq\left(2.82 \times 10^{-21}\right) \cdot\left(\frac{1 H z}{f}\right)
$$

These values of strain, the numerical count, and also of $n_{f}$ give a bit count and entropy which will lead to possible limits as to how much information is trans ferred. Note that the beginning of relic inflation $\lambda_{G W}$ $\sim 10^{-1}$ meters $\Rightarrow n_{f} \propto 10^{6}$ graviton / unit-phase-space

for $f_{G W} \sim 10^{9}$ Hertz This is to have, say a starting point in pre inflationary physics of $f_{G W} \sim 10^{22}$ Hertz when $\lambda_{G W} \sim 10^{-14}$ meters, i.e. a change of $\sim 10^{13}$ orders of magnitude in about $10^{-25}$ seconds, or less.

\section{3. $1^{\text {st }}$ Part of Conclusion. Can We Justify/}

\section{Isolate out an Appropriate $T^{u v}$ if one has Non Zero Graviton Rest Mass?}

It is difficult. It depends upon understanding what is meant by emergent structure, we assert that the mathematical self organized criticality structure is akin to a definition as to how Dp branes arise at the start of inflation. What is the emergent structure permitting $\oint p_{i} d x_{k}=\hbar \delta_{i, k}$ to hold? [1] What is the self organized criticality structure leading to forming an appropriate (1) $T^{u v}$ if one has non zero graviton rest mass? Answering such questions will permit us to understand how to link 
finding $T^{(1)}$ in a GW detector, its full analytical linkage to $\beta$ in Equation (16). The following construction is used to elucidate how a EM Gaussian sense beam can perhaps be used to eventually help in isolating $T^{u v}$ in a GW detector. This construction below is to be used to investigate 'massive gravitons'/and also the initial structure of self organized criticality, in the aftermath of graviton/gravitational wave generation.

\section{1. $2^{\text {nd }}$ Part of Conclusion: In Terms of the Planckian Evolution and a Pre Planckian Space Time}

We wish to summarize what we have presented in an orderly fashion. Doing so is a way of stating that Analog, (Pre Ocononian) reality is the driving force behind the evolution of inflationary physics

1) Pre Octonian gravity physics (analog regime of reality) features a break down of the Octonian gravity commutation relationships when one has curved space time. This corresponds, as brought up in the Jacobi iterated mapping for the evolution of degrees of freedom to a build up of temperature for an increase in degrees of freedom from 2 to over 1000. Per unit volume of space time. The peak regime of where the degrees of freedom maximize is where the Octonian regime holds.

2) Analog physics, prior to the build up of temperature can be represented by Equation (7) and Equation (8). The first of these mappings is an ergotic mapping, a perfect mixing regime from many universes into our own present universe. This mapping requires a deterministic quantum limit as similar to what t'Hooft included in his embedding of Quantum physics in a larger, non linear theory [11].

This will help to localize the regime of space time build up potentially giving experimental details to the formation of Equation (14) and Equation (15) above. The claim is that the physical evolution described by Equation (7) and Equation (8) would arise, as a dynamic evolutionary process in the Micro physics regime leading to an embedding of quantum physics, in a larger, non linear theory, which would lead to massive gravitons being synthesized whole scale. This whole scale synthesis of gravitons with a small rest mass occurs in initial micro physics background as indicated above, a result we hope to confirm via accurate measurements of the phase.

\section{Acknowledgements}

This work is supported in part by National Nature Science Foundation of China grant No. 11075224 Dr Fangyu Li of Chongquing University gave extensive technical assistance to the author as to understanding the phase change in gravitational waves due to early universe cosmology. Stuart Allen gave significant encouragement to the author as to finalizing this document for publication.

\section{References}

[1] L. Crowell, Quantum Fluctuations of Space-time, in World Scientific Series in Contemporary Chemical Physics, Volume 25, Singapore, Republic of Singapore, 2005.

[2] A. W. Beckwith, "Identifying a Kaluza- Klein treatment of a Graviton permitting a de-celeration parameter $\mathrm{Q}(\mathrm{Z})$ as an alternative to standard DE “,Journal of Cosmology, Volume 13, 2011, http://journalofcosmology/BeckwithGraviton.pdf

[3] F. Li, and N. Yang, "Phase and Polarization State of High Frequency Gravitational waves", Chin Phys. Lett. Vol 236, No 5(2009), 050402, pp 1-4

[4] F. Li,. M.,Tang, D. Shi, "Electromagnetic response of a Gaussian beam to high frequency relic gravitational waves in quintessential inflationary models", PRD 67, 104008 (2003), pp1-17

[5] S. Chaturvedi, "Mutually Unbiased Bases", Pramana Journal of Physics, Vol. 52, No. 2, pp 345-350

[6] John C. Baez, "Renyi Entropy and Free Energy", http://arxiv.org/abs/1102.2098

[7] A. W. Beckwith, arXiv:0804.0196 [physics.gen-ph], AIP Conf.Proc. 969:1018-1026, 2008

[8] D. K., Park, H. Kim, H., and S. Tamarayan, "Nonvanishing Cosmological Constant of Flat Universe in Brane world Senarios," Phys. Lett. B 535, 5-10 (2002).

[9] A. W. Beckwith," How to Use the Cosmological Schwinger Principle for Energy Entropy, and "atoms of Space-Time" to Create a Thermodynamic Space-Time and Multiverse"; accepted for the DICE 2010 proceedings; http://vixra.org/abs/1101.0024, 2010

[10] M. Maggiorie,. "Gravitational Wave Experiments and Early Universe Cosmology”, Physics Reports 331 (2000) pp. 283-367

[11] G. t' Hooft, Beyond the Quantum, edited by Th. M. Nieuwenhuizen et al. Singapore, World Press Scientific, 2006; http://arxiv.org/PS_cache/quant-ph/pdf/0604/0604008v2. pdf. 